\title{
A "hotspot" for autoimmune T cells in type 1 diabetes
}

\author{
Brian D. Stadinski, ${ }^{1}$ Reinhard Obst, ${ }^{2}$ and Eric S. Huseby ${ }^{1}$ \\ 'Department of Pathology, University of Massachusetts Medical School, Worcester, Massachusetts, USA. ${ }^{2}$ nstitute for Immunology, Ludwig-Maximilians-Universität Munich, Munich, Germany.
}

\begin{abstract}
The ability of a single T cell antigen receptor (TCR) to cross-react with multiple antigens allows the finite number of $T$ cells within an organism to respond to the compendium of pathogen challenges faced during a lifetime. Effective immune surveillance, however, comes at a price. TCR crossreactivity can allow molecular mimics to spuriously activate autoimmune T cells; it also underlies $\mathrm{T}$ cell rejection of organ transplants and drives graft-versus-host disease. In this issue of the $J C l$, Cole and colleagues provide insight into how an insulin-reactive $T$ cell cross-reacts with pathogen-derived antigens by focusing on a limited portion of the peptides to provide a hotspot for binding. These findings dovetail with recent studies of alloreactive and autoimmune TCRs and suggest that the biochemical principles that govern conventional protein-protein interactions may allow the specificity and cross-reactivity profiles of $\mathrm{T}$ cells to be predicted.
\end{abstract}

\section{Introduction}

T cells constantly survey cells of the body for signs of pathogen infection and tumorigenesis. The recognition of infection or altered self is achieved through $\mathrm{T}$ cell antigen receptor binding (TCR binding) to pathogen- or tumor-derived peptides of 8-15 amino acids in length that are presented by host-derived major histocompatibility complex proteins (MHC proteins). The seemingly endless breadth of antigenic coverage is predicated on the mature $\mathrm{T}$ cell repertoire comprising millions of unique clonotypes. Every individual $\mathrm{T}$ cell expresses a unique TCR that is formed through somatic recombination of $\mathrm{V}(\mathrm{D}) \mathrm{J}$ gene segments and nucleotide additions, as well as pairing of the randomly created TCR $\alpha$ and TCR $\beta$ chains. Though the clonal complexity of mature T cell repertoires is large, it would be vastly insufficient to provide complete pathogen coverage if a given $\mathrm{T}$ cell clonotype could only recognize a single peptide sequence presented by MHC molecules. For example, there are approximately $5 \times 10^{11}$ possible sequence combinations for a 9 amino acidlong peptide. Mature T cells are therefore equipped with TCRs that can recognize multiple distinct peptides, thus avoiding holes in the repertoire $(1,2)$.

The rate at which $\mathrm{T}$ cells can recognize disparate peptide-MHC (pMHC) ligands is determined during development as the result of Darwinian forces acting on immature $\mathrm{T}$ cells (thymocytes) following the initial expression of their TCR. There are perhaps 10,000 unique self-peptides that are presented by MHC molecules within the thymus. Failure to recognize any of these self-peptides at a weak but appreciable level will arrest the thymocyte maturation process. This developmental checkpoint puts a lower limit on the rate of peptide cross-reactivity, as TCRs have to engage at least 1/10,000 pMHC ligands with measurable binding affinity. Conversely, thymocytes that expresses TCRs that are unable to sufficiently distinguish different self-peptides or recognize any self-peptides with a strong affinity are signaled to undergo apoptosis $(1,2)$. The fittest $\mathrm{T}$ cells - those that are most likely to provide broad immunological coverage while limiting overt self-reactivity - are ushered along the developmental pathway and are

Related Article: p. 2191

Conflict of interest: E.S. Huseby received research funding from EMD Serono.

Reference information: / Clin Invest. 2016;126(6):2040-2042. doi:10.1172/JCI88165.

ultimately exported to become part of the mature $\mathrm{T}$ cell repertoire. Unfortunately, $\mathrm{T}$ cell development is not perfect, and some autoreactive $\mathrm{T}$ cells slip through this culling process.

\section{TCRs have different rates of cross-reactivity}

Studies of the reactivity patterns of $\mathrm{T}$ cells toward cells expressing allogeneic MHC revealed the first clues about TCR-recognition characteristics. It was determined that MHC allotypes can differ by more than 30 amino acids - many, but not all, of which affect the binding requirements of the peptides presented. Two models were proposed to explain $\mathrm{T}$ cell recognition of allo-pMHC. One posited that alloreactive $\mathrm{T}$ cells focus on peptides that are associated with the foreign MHC due to its different peptide cargo and are thus allorestricted (3). The second model suggests that the alloreactive $\mathrm{T}$ cells focus on the differences between the host and alloMHC molecules themselves (4). Evidence to support both models arose (5), and sorting out why different types of recognition patterns arise required the use of combinatorial peptide libraries to probe the specificity requirements of allo-MHC responsive $\mathrm{T}$ cell repertoires. $\mathrm{T}$ cells that respond to foreign MHC alleles that are the most divergent from the MHC of the host are the least dependent upon the bound peptide and instead target MHC residues. In contrast, $\mathrm{T}$ cells that are reactive to $\mathrm{MHC}$ alleles that differ only at 1-3 positions (and are thus micropolymorphic in comparison) are similarly dependent on the bound peptide, as are conventional, host-MHCrestricted $\mathrm{T}$ cells (6). Though relatively little is known about how the MHC-centered part of alloreactivity to macropolymorphic MHC differences operates on a molecular level $(5,7)$, these findings already argued that the selective forces shaping the $\mathrm{T}$ cell repertoire heightens the sensitivity toward the recognition of peptides presented by self- and similar-to-self MHC alleles.

As the biochemistry of TCR recognition continues to be probed, and the 


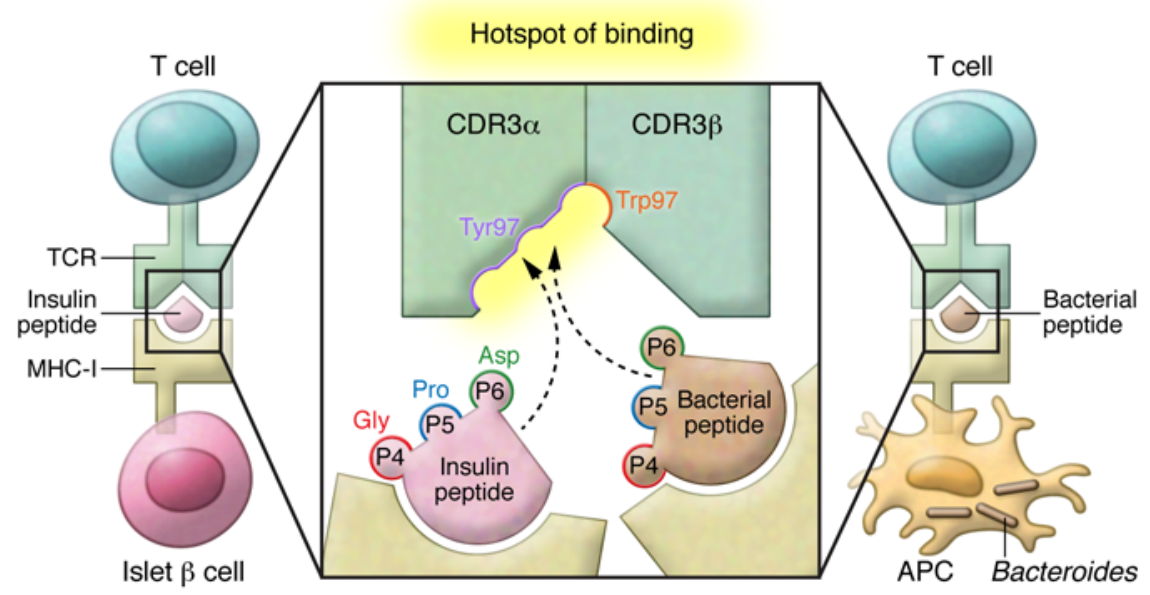

structural database of TCR-pMHC complexes expands, molecular insights that explain particular pMHC cross-reactivity patterns are giving way to common themes. Despite the enormous amount of variability in TCRs and pMHC ligands, these interactions are governed by the same biochemical principles as other protein-protein interactions. A common feature among receptor-ligand binding sites is a hydrophobic core that is created through the exclusion of water (the hydrophobic effect), which provides a large amount of binding strength (8). The location of strong-affinity interactions, and the amino acids that comprise it, is often referred to as a "hotspot" of binding energy (9). During T cell ligand recognition, these hot spots can incorporate TCRMHC interactions, as well as TCR-peptide interactions (10-12). TCR-pMHC contacts that occur peripherally to the hotspots can enhance the specificity of the interaction by providing additional binding affinity or by creating steric hindrance that disrupts the binding reaction (13). Because the amino acids that compose the hotspots of binding are of central importance, the number of amino acids involved and their location (peptide versus MHC residues) will have a critical role in defining the specificity of $\mathrm{T}$ cells. For example, highly self-reactive and alloreactive TCRs have been found to predominately create a TCR-MHC binding hotspot and, thus, are less dependent upon TCR-peptide interactions. In contrast, self-tolerant, nonalloreactive, or weakly alloreactive TCRs create strong-affinity binding with peptide residues $(14,15)$.

\section{Autoimmune TCRs bind self- and foreign ligands using the same hotspots}

In this issue, Cole, Bulek, and colleagues report on how molecular mimics may activate human insulin-reactive $\mathrm{CD} 8^{+} \mathrm{T}$ cells (16). The $1 \mathrm{E} 6 \mathrm{~T}$ cell was originally isolated from the peripheral blood of a patient with recent onset type 1 diabetes. This $\mathrm{T}$ cell clone is cytotoxic and kills pancreatic islet $\beta$ cells in vitro through recognition of a peptide derived from the preproinsulin signal sequence. Cole et al. screened a combinatorial peptide library and identified a panel of molecular mimics, including peptides carried by the human pathogens Bacteroides fragilis and Clostridium asparagiforme. Using a combination of structural and biochemical studies, the authors demonstrated how the 1E6 TCR binds seven different pMHC ligands. Remarkably, all seven binding reactions used the same hotspot of binding that formed between the TCR CDR3 residues Tyr97a and $\operatorname{Trp} 97 \beta$ and the peptide position 4, 5, and 6 (P4, P5, P6) residues Gly-Pro-Asp, which are present within insulin and each of the molecular mimics (Figure 1). These contacts ultimately were shown to account for approximately half of the TCR-pMHC binding site. Peripheral to this conserved hotspot of binding, the TCR-pMHC interactions present within each structure were distinct. Moreover, these contacts impact the overall affinity and thermodynamic signature of the TCR-pMHC interaction but are replaceable with different combinations of amino acids. Thus, the rate of cross-reactivity for this autoimmune $\mathrm{T}$ cell clone is primarily set by the frequency
Figure 1. A focused binding hotspot explains how $T$ cells harboring an insulin-targeting TCR can become activated. The 1E6 T cell was derived from an individual with type 1 diabetes and recognizes an insulin peptide. In this issue, Cole, Bulek, and colleagues reveal that the TCR of the 1E6 T cell also recognized molecular mimics derived from bacteria, such as species of Bacteroides. Further characterization of these interactions revealed that the 1E6 TCR binds multiple ligands that use the same hotspot of binding (see insert) that forms between TCR residues (Tyr97 of CDR3 $\alpha$ and Trp97 of CDR3 $\beta$ ) and Gly, Pro, and Asp residues within the insulin peptide and the molecular mimics at $\mathrm{P} 4, \mathrm{P} 5$, and P6 (see insert).

at which peptides carry the Gly-Pro-Asp sequence or carry chemically similar amino acids in their stead.

\section{Conclusions and future directions}

How generalizable might the use of focused hotspots be for allowing autoimmune $\mathrm{T}$ cells to be activated by molecular mimics? Pioneering studies by Wucherpfennig and colleagues (17) demonstrated that myelin basic protein-specific (MBP-specific) $\mathrm{T}$ cells isolated from multiple sclerosis patients can be activated by some pathogen-derived peptides that carry a His-Phe motif at central residues of the peptide. A detailed analysis of one of these $\mathrm{T}$ cells, Hy.1B11 TCR, shows that a single CDR3 $\alpha$ residue, F95, makes the majority of contacts with both an MBP peptide and two microbial peptides, focusing on the peptide P2 His (18). Such TCR-pMHC interactions have been observed in allogeneic settings. For example, the LC13 TCR interactions target the $\mathrm{P} 6-\mathrm{P} 8$ region of the peptide and, in particular, the aromatic residue at P7 that is present in viral, allo-, and mimetope peptides (19). Conversely, the alloreactive 4F23 TCR utilizes the same CDR3 $\beta$ residues to contact hydrophobic residues at $\mathrm{P} 7$ of divergent peptide ligands (20). In each of these cases, the hotspot of interaction is focused on the use of particular CDR3 residues that contact distinct biochemically homologous regions across the different peptides.

The elucidation of so-called rules of engagement that would allow prediction of whether a $T$ cell will functionally engage particular ligands based on the sequence 
of the TCR is still a ways off. Nevertheless, biochemical and structural analyses are beginning to reveal common strategies used by TCRs to bind pMHC and control the rate of cross-reactivity. Focused hotspots of binding are central to these interactions (21) and are often created by the hydrophobic effect. This attribute of TCR-pMHC interactions, highlighted in the structures by Cole et al., leads to a clear hypothesis: TCRs carrying amino acids that promote hotspot formation (such as aromatic residues) within their antigen binding site should have an increased propensity to engage $\mathrm{pMHC}$ ligands. Likewise, peptides that carry these same residues at TCR contact positions should be more efficient at eliciting $\mathrm{T}$ cell responses.

\section{Acknowledgments}

This work was funded in part by grants from the NIH/NIDDK (5R01DK095077-04) and the NIH/NIAID (U19 AI109858) to E.S. Huseby and from the German Research Council (SFB1054-B07) to R. Obst.

Address correspondence to: Eric S. Huseby, Department of Pathology, University of Massachusetts Medical School, AS9-1055 Sherman Center, 368 Plantation Street, Worcester, Massachusetts, USA. Phone: 508.856.2180; E-mail: Eric. Huseby@umassmed.edu.
1. Hogquist KA, Jameson SC. The self-obsession of T cells: how TCR signaling thresholds affect fate 'decisions' and effector function. Nat Immunol. 2014;15(9):815-823.

2. Vrisekoop N, Monteiro JP, Mandl JN, Germain RN. Revisiting thymic positive selection and the mature $\mathrm{T}$ cell repertoire for antigen. Immunity. 2014;41(2):181-190.

3. Matzinger P, Bevan MJ. Hypothesis: why do so many lymphocytes respond to major histocompatibility antigens? Cell Immunol. 1977;29(1):1-5

4. Bevan MJ. High determinant density may explain the phenomenon of alloreactivity. Immunol Today. 1984;5(5):128-130.

5. Felix NJ, Allen PM. Specificity of T-cell alloreactivity. Nat Rev Immunol. 2007;7(12):942-953.

6. Obst R, Netuschil N, Klopfer K, Stevanovic S, Rammensee HG. The role of peptides in T cell alloreactivity is determined by self-major histocompatibility complex molecules. J Exp Med. 2000;191(5):805-812.

7. Rossjohn J, Gras S, Miles JJ, Turner SJ, Godfrey DI, McCluskey J. T cell antigen receptor recognition of antigen-presenting molecules. Annu Rev Immunol. 2015;33:169-200.

8. Chandler D. Interfaces and the driving force of hydrophobic assembly. Nature. 2005;437(7059):640-647.

9. Clackson T, Wells JA. A hot spot of binding energy in a hormone-receptor interface. Science. 1995;267(5196):383-386

10. Baker BM, Turner RV, Gagnon SJ, Wiley DC, Biddison WE. Identification of a crucial energetic footprint on the $\alpha 1$ helix of human histocompatibility leukocyte antigen (HLA)-A2 that provides functional interactions for recognition by tax peptide/HLA-A2-specific T cell receptors. J Exp Med. 2001;193(5):551-562.

11. Wu LC, Tuot DS, Lyons DS, Garcia KC, Davis MM. Two-step binding mechanism for T-cell receptor recognition of peptide MHC. Nature. 2002;418(6897):552-556.

12. Borg NA, et al. The CDR3 regions of an immunodominant $\mathrm{T}$ cell receptor dictate the 'energetic landscape' of peptide-MHC recognition. Nat Immunol. 2005;6(2):171-180.

13. Huseby ES, Crawford F, White J, Marrack P, Kappler JW. Interface-disrupting amino acids establish specificity between $\mathrm{T}$ cell receptors and complexes of major histocompatibility complex and peptide. Nat Immunol. 2006;7(11):1191-1199.

14. Dai S, et al. Crossreactive T cells spotlight the germline rules for $\alpha \beta$ T cell-receptor interactions with $\mathrm{MHC}$ molecules. Immunity. 2008;28(3):324-334.

15. Stadinski BD, et al. A role for differential variable gene pairing in creating $\mathrm{T}$ cell receptors specific for unique major histocompatibility ligands. Immunity. 2011;35(5):694-704.

16. Cole DK, et al. Hotspot autoimmune T cell receptor binding underlies pathogen and insulin peptide cross-reactivity. J Clin Invest. 2016;126(6):2191-2204

17. Wucherpfennig KW, Strominger JL. Molecular mimicry in T cell-mediated autoimmunity: viral peptides activate human $\mathrm{T}$ cell clones specific for myelin basic protein. Cell. 1995;80(5):695-705.

18. Sethi DK, Gordo S, Schubert DA, Wucherpfennig KW. Crossreactivity of a human autoimmune TCR is dominated by a single TCR loop. Nat Commun. 2013;4:2623.

19. Macdonald WA, et al. T cell allorecognition via molecular mimicry. Immunity. 2009;31(6):897-908.

20. Adams JJ, et al. Structural interplay between germline interactions and adaptive recognition determines the bandwidth of TCR-peptide-MHC cross-reactivity. Nat Immunol. 2016;17(1):87-94.

21. Bogan AA, Thorn KS. Anatomy of hot spots in protein interfaces. J Mol Biol. 1998;280(1):1-9. 